\title{
Novel Design of a Highly Sensitive RF Strain Transducer for Passive and Remote Sensing in Two Dimensions
}

\author{
Trang T. Thai, Student Member, IEEE, Herve Aubert, Senior Member, IEEE, Patrick Pons, \\ Gerald DeJean, Member, IEEE, Manos M.Tentzeris, Fellow, IEEE, and Robert Plana, Senior Member, IEEE
}

\begin{abstract}
A novel design of a highly sensitive wireless passive RF strain transducer is presented based on a patch antenna loaded with an open loop that is capable of sensing strain independently in two directions. An original idea of utilizing a cantilever at the gap of the open loop significantly improves the sensitivity of resonant frequency shifts. The frequency shifts in two distinct resonant modes are detected based on two dominant orthogonal modes of the patch resonators. In measurements, the prototypes achieved a sensitivity of $2.35 \%$ frequency shift per $1 \%$ strain, more than twice that of existing strain transducers of the same class. In simulations, the new design achieved a theoretical sensitivity up to four times as high as existing designs of RF passive wireless strain transducers. The ground plane allows for the sensitivity of the sensor to be independent from the applied surface. An implementation example of the passive remote sensing system based on the proposed strain transducer is also discussed as a proof-of-concept case. Based on calculations, the interrogation method in the example shows a radar cross section fluctuation of $3.8 \mathrm{~dB}$ corresponding to the strain induced at the sensor.
\end{abstract}

Index Terms-Passive sensing, remote sensing, RF transducer, strain stress sensor, wireless sensor.

\section{INTRODUCTION}

A CRUCIAL factor in many industries ranging from civil infrastructure and mechanical equipment to aerospace and various medical applications is structural health. In measuring the structural health, strain sensing has been the key parameter to monitor civil structures and to address the safety assurance of roads, bridges, and building supports in order to avoid unexpected collapses [1]. Strain is a parameter that indicates a physical deformation and mechanical loading. In manufacturing processes and constructions, strain sensing allows the monitoring of vibration, excessive loading, and

Manuscript received December 14, 2012; revised January 16, 2013; accepted January 16, 2013. Date of publication February 13, 2013; date of current version March 07, 2013.

T. T. Thai and M. M. Tentzeris are with the School of Electrical and Computer Engineering, Georgia Institute of Technology, Atlanta, GA 30332 USA (e-mail: trang.thai@gatech.edu; etentze@ece.gatech.edu).

H. Aubert and R. Plana are with UPS, INSA, INP, ISAE, UT1, UTM, LAAS, Université de Toulouse, F-31077 Toulouse Cedex 4, France (e-mail: herve.aubert@enseeiht.fr; plana@laas.fr).

P. Pons is with CNRS-LAAS, F-31077 Toulouse Cedex 4, France (e-mail:pons@laas.fr).

G. R. DeJean is with Microsoft Research, Redmond, WA 98052 USA (e-mail: dejean@microsoft.com).

Color versions of one or more of the figures in this paper are available online at http://ieeexplore.ieee.org.

Digital Object Identifier 10.1109/TMTT.2013.2243751 crack developments to be detected early [2], [3]. In medical uses, strain sensing also include implantable sensors for bone, joint healing processes, and bone fracture monitoring [4], [5]. In aerospace, strain is especially sensitive for operations of aircraft, including the conditions of wings and blades, and the fatigue of the aircraft body [6]. Therefore, strain sensing needs to be accurate, quick, and efficient to allow long-term monitoring with little maintenance for public safety and economic and industrial prosperity. However, uses of sensors embedded in structures and machines remain limited.

Strain sensors have been widely developed utilizing different techniques; the most common types are resistive and capacitive gage. Since those sensors typically require wiring and/or complex circuits, they add a weight overhead [7], which is highly undesirable, especially for the implementation on aircraft wings or helicopter blades. Besides those wired sensors, some wireless sensors have been reported [8], [9]. They usually include an embedded microprocessor and an RF module, which is integrated with the transducer. Such a circuit requires battery power to operate, has a limited lifetime, and requires more frequent maintenance and replacement. Thus, they are unsuitable for long-term monitoring. Other sensing techniques based on optical fibers are compact, lightweight, and capable to be sensed remotely, but expensive [6]-[10]. Therefore, wireless passive strain sensors that can address the problems mentioned earlier are highly desirable.

Recently, through a number of works that address the sensing of numerous physical parameters, such as pressure, temperature, and strain, the concept of RF transducers has been introduced [11]-[22]. An RF transducer is a structure that can transform a physical parameter into an RF parameter, such as a resonant frequency shift, a radiation pattern, or a radar cross section (RCS) level change that is readily detectable by a remote system. Ideally, such sensors should simultaneously be the resonant radiators, which can eliminate the need for additional antennas, which are the major limiting factor in miniaturization of these devices. In the past, rectangular and circular patch antennas have been utilized [17]-[19] that operate based on frequency shifts. However, their sensitivity is limited. One reported design is based on split-ring resonators that constitute a resonant scatterer whose resonant frequencies are sensitive to strain [20]. However, it is highly dependent on the applied surface, and it has low sensitivity. In previous work, a design was introduced that is based on a patch antenna loaded with an open loop [21], [22]. It could achieve significantly higher sensitivity than previous RF strain transducers. In this extended work, we further 


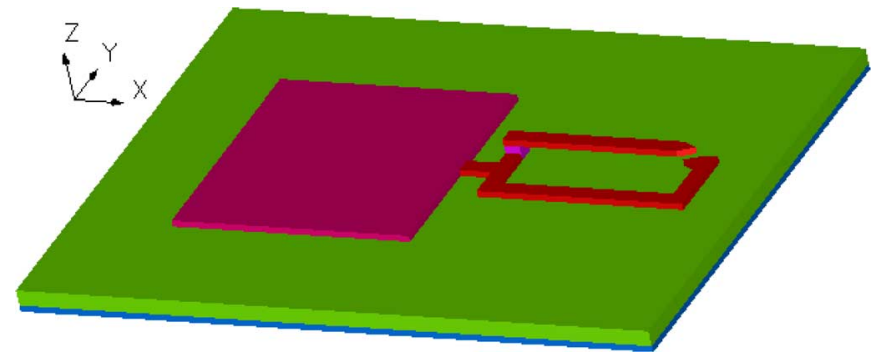

Fig. 1. 3-D view of the single-loop loaded strain transducer.

enhance the performance of this sensor and report for the first time the capability of sensing strain in two independent directions, i.e., enabling sensing of arbitrary on-plane strain direction in a passive and remote platform. To the best knowledge of the authors, this transducer is the first of its kind in structural health monitoring. This paper is presented in the following order. In Section II, we discuss the designs and principles of operation. Section III illustrates how the sensor designs are modeled with regard to sensing strain and also reports the simulation results. Section IV shows benchmarking prototypes and the strain measurement setup followed by results and discussions in Section V. Section VI illustrates a possible remote sensing scheme based on frequency-modulated continuous-wave (FWCW) radar.

\section{Design AND PRINCIPLES OF OPERATION}

The single-loop structure of the new strain sensor in 3-D view is shown in Fig. 1 [21], [22], while the 2-D strain transducer design over the sensing plane is shown in Fig. 2. In the 2-D design, a rectangular patch is loaded with two stubs, each on the radiating edge of the corresponding resonant mode, i.e., two dominant orthogonal modes, induced by orthogonal excitations that affect two different sides of the patch. Each stub in turn is connected to an open loop whose resonant frequency depends on the open gap capacitance. Specifically, an open loop is loaded along $x$ in order to modify the dominant resonant mode that is induced by the $x$-polarization excitation. Similarly, another open loop is loaded along $y$ in order to modify the dominant resonant mode that is induced by the $y$-polarization excitation. The lowest resonant frequency of the open loop, determined by the length of the loop and the gap capacitance, is designed to be close to the dominant resonant frequency of the patch along the $x$ - or $y$-direction (Fig. 2). As a result, for each orthogonal mode of the patch, the structure generates a dual frequency response in which the separation of the two frequencies is strongly influenced by the capacitance of the open ring. The correspondence implies that the resonant peaks, especially the one controlled by the open loop, are very sensitive to the capacitance value of the gap for each polarization mode. The open loops here are modified from a traditional open loop, which is the key idea first introduced in our previous work [21], [22] on sensitivity improvement. This is the first of the two operational principles of the new 2-D strain transducer proposed in this work.

Strain is measured by the deformation of the material volume along the strain direction. It is denoted as $\varepsilon_{\mathbf{L}}=\Delta L / L$. The "zero-strain" length $L_{o}$ in the stretched direction is deformed

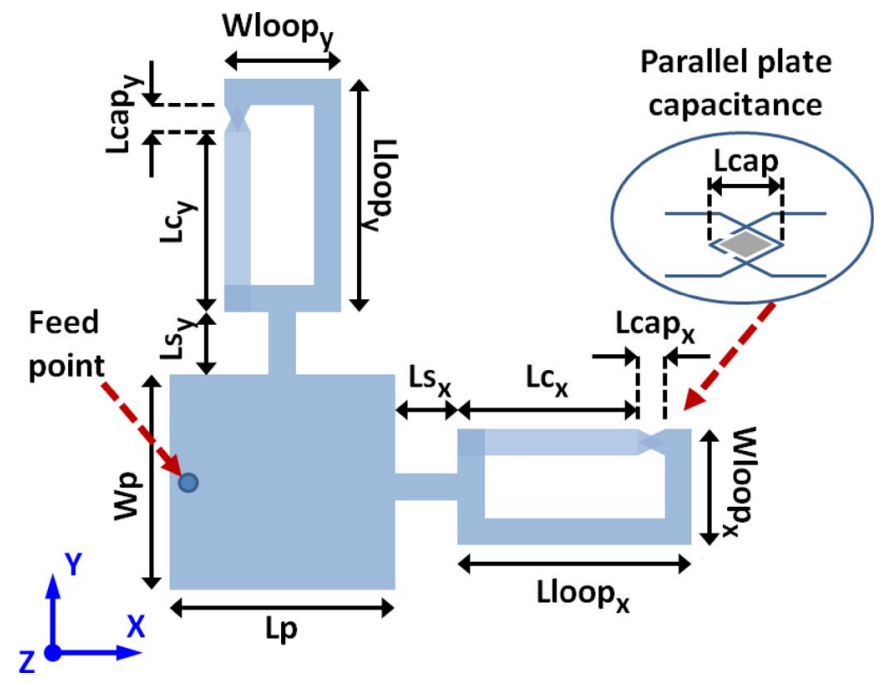

Fig. 2. Top view of the new design of the strain sensor and zoom-in view of the capacitance gap on the open ring.

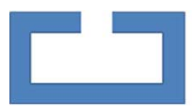

(a)

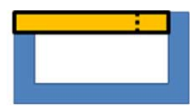

(b)
Fig. 3. Top view of the traditional and modified loops. (a) Traditional open loop. (b) Modified open loop.

into $L$, as shown in (1) Strain is unitless, and usually measured in $\%$ or micro-strain $(\mu \varepsilon)$, which is $10^{-3}$ of $\Delta \mathrm{L} / \mathrm{L}$

$$
L=\left(1+\varepsilon_{L}\right) L_{0} .
$$

Therefore, any resonant structure attempting to transform this change directly into a detectable RF signal is inherently limited as the existing designs have shown [17]-[20]. As illustrated in [21] and [22], to solve this problem, we utilize a cantilever implemented on each open loop loaded on a patch antenna instead of the patch itself solely as done in [17] and [18].

Each cantilever is shorted electrically and fixed mechanically at one end of the corresponding open loop, while the free end of the cantilever and the other end of the open loop form a parallel plate capacitor. Thus, the split gap between the two ends of the traditional open ring [see Fig. 3(a)] is transformed into the separation gap of a parallel plate capacitor (Figs. 3(b) and 4). The transformation from the configuration of Fig. 3(a) into that of Fig. 3(b) allows the capacitance to be tuned nonlinearly with respect to the surface strain in the following mechanism. As a certain $k \%$ strain is induced on the substrate along the $x$-direction (Fig. 4), the $L$ gap is increased by $\Delta L$ gap, which is equal to $k \% \times L$ gap. Since the cantilever is fixed to the surface only at one end, it remains unaffected by this strain, and thus, its length, $L c$, is constant. Therefore, the entire length deformation $\Delta L$ gap is transferred to the change in $L$ cap (Fig. 4). Consequently, the capacitance is modified by $k \% \times L$ gap instead of $k \% \times L$ cap, in which $L$ gap can be engineered to be significantly larger than Lcap, as shown in Fig. 2. The use of triangular tips at the loop gap further increases the rate of change of the capacitance with a given rate of change of the $L$ cap induced by a given strain. 


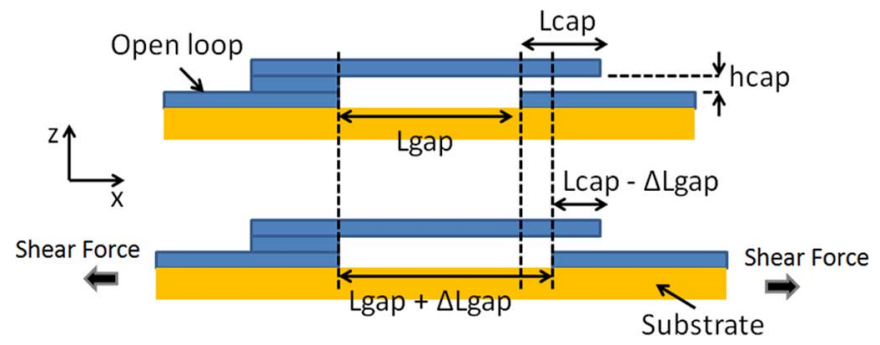

Fig. 4. Cross-section view of a cantilever implemented on a modified loop loaded in the new strain sensor.

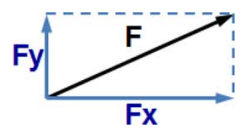

Fig. 5. Illustration of a force in an arbitrary direction decomposed into orthogonal components.

The two inherent independent polarizations of a patch resonator allow independent interrogation of strain in two orthogonal directions, and the strain sensing in one direction is not influenced by the strain in the other. The operation of the strain sensor shown in Fig. 2 is clarified in Section III. This operational principle lays the foundation for strain sensing in arbitrary directions because of the shear force (applied on the material surface and responsible for the surface deformation) can be decomposed into two orthogonal components (Fig. 5). This is the second of the two operational principles of the new 2-D strain transducer proposed in this work.

\section{Modeling AND Simulations}

\section{A. Modeling}

As a proof of concept for the above design, a transducer is designed to operate around $3 \mathrm{GHz}$ on Kapton substrate of $100-\mu \mathrm{m}$ thickness with a dielectric constant of 3.4. An increase of $1 \%$ is applied to each length of the parameters along the $x$-direction only, then RF responses for each polarization of the patch-based transducer are analyzed in order to show the capability of 2-D sensing of this design. The applied strain in the $x$-direction increases the length of the parameters $L p, L s_{x}$, and $L \operatorname{loop}_{x}$. Note that $L \operatorname{loop}_{x}$ increased by $1 \%$, while $L c_{x}$ is fixed. Consequently, the dimension $L$ cap $x$ decreases by $1 \%$ of $L \operatorname{loop}_{x}$. Additionally, the substrate under strain also experiences the Poisson effect, which essentially causes a contraction in the direction transverse to the strain. Thus, the dimension $W p$ effectively decreases. The Poisson effect is the ratio of this contraction to the extension caused by the strain. The Poisson effect can be calculated as shown in (2), in which $w$ denotes the width of the dimension that is transverse to the strain direction, $v_{p}$ is Poisson's ratio of the Kapton, and $\varepsilon_{L}$ is the applied strain. Other parameters along the $x$-direction, such as $W \operatorname{loop}_{y}$, and those under the Poisson effect, such as the thickness of the substrate and the width of the open loop, are negligible to the RF response of the structure; hence, they are not accounted for. This simplification is justified in the measurement results shown in Section V. In addition, within a few percent $(1 \%-5 \%)$ of the loop width $W$ loop,

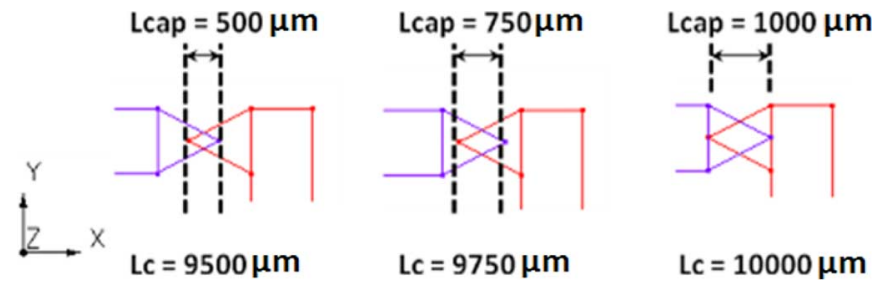

Fig. 6. Different configurations of the tip capacitance as different $L c$ values, the length of the cantilever, are implemented.

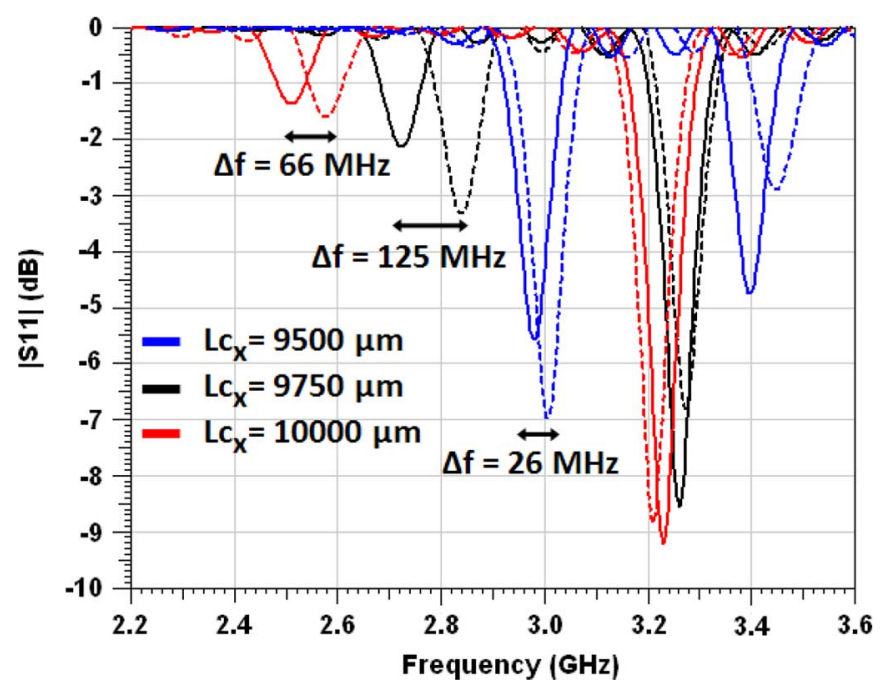

Fig. 7. Different configurations of the tip capacitance as different $L c$ values are implemented. The solid lines represent nonstrain conditions, and the slash lines represent $1 \%$ strain conditions.

the resonant frequency of the open loop is found to be only negligibly influenced

$$
w=\left(1+v_{p} \varepsilon_{L}\right) w_{0} .
$$

\section{B. Study of the First Principle of Operation-Modification of the Open Loop for High Sensitivity}

Different $L c_{x}$ values are simulated on the single-loop patch structure (Fig. 1) to investigate the effect of the loop gap capacitance upon the resonant frequencies of the loop-loaded patch. For simplicity and without loss of accuracy, we have not included the second loop that is loaded along the $y$-direction in order to analyze the effect of the loop gap capacitance. In this study, we investigated the first principle of operation of the transducer for only the $x$-polarization mode of the patch. We will show later that this approach is sufficient to address the frequency shifts that occur in the 2-D structure in practical measurement (Fig. 2). We varied only $L c_{x}$ in these simulations while keeping the other values the same for each $L c_{x}$ value in both nonstrain and strain-load conditions. Different $L c_{x}$ values effectively yield different $L \operatorname{cap}_{x}$ values, as illustrated in Fig. 6. $S 11$ simulations results are presented in Fig. 7 for $L c_{x}=9500,9750$, and $10000 \mu \mathrm{m}$. Each $L c_{x}$ value with either zero or $1 \%$ strain conditions is simulated using the parameters provided in Table I. Notice that the gap between the two parallel surfaces, the lower face of the cantilever free end and the upper 
TABLE I

SUMMARY OF SIMULATION PARAMETERS

\begin{tabular}{|l|c|c|}
\hline Dimensions $(\mu \mathrm{m})$ & No strain & Strain $1 \%$ \\
\hline $\mathrm{Lp}$ & 26000 & 26260 \\
\hline $\mathrm{Wp}$ & 25000 & 24925 \\
\hline $\mathrm{Ls}_{\mathbf{x}}$ & 2000 & 2020 \\
\hline Lloop $_{\mathbf{x}}$ & 13000 & 13130 \\
\hline Wloop $_{\mathbf{x}}$ & 5000 & 5000 \\
\hline Ls $_{\mathbf{y}}$ & 2000 & 2000 \\
\hline Lloop $_{\mathbf{y}}$ & 13000 & 13000 \\
\hline Wloop & 5000 & 5000 \\
\hline c (width of stub and loop) & 1000 & 1000 \\
\hline hsub (substrate thickness) & 100 & 100 \\
\hline hcap (gap between the cantilever and loop) & 10 & 10 \\
\hline
\end{tabular}

face of the open-ended loop, is set to $10 \mu \mathrm{m}$. This parameter is highly critical to the sensitivity of the strain transducer because it determines the initial gap capacitance as well as the value of $\operatorname{Lloop}_{x}$.

It is observed from the $|S 11|$ results in Fig. 7 that each model has a dual frequency response with two close resonant frequencies. The sensitivity of the transducer depends on the value of $L c_{x}$, which determines the initial capacitance and rate of change of the capacitance as $L c_{c a p}$ is decreased. Here, the highest sensitivity that can be achieved is found to be for $L c_{x}=9750 \mu \mathrm{m}$, which induces a frequency shift of $125 \mathrm{MHz}$ at $2.72 \mathrm{GHz}$, or $4.6 \%$ frequency shift per $1 \%$ strain, as reported in [21] and [22]. This sensitivity is more than four times the sensitivity achieved by rectangular and circular patch antennas previously proposed that rely only on the change in the dimensions of the patches and achieved a sensitivity of only $0.9 \%-1.0 \%$ frequency shift per $1 \%$ strain [17]-[19]. It is observed that as the strain is applied, the resonant frequencies increased due to the resulting decreased overall capacitance. Such responses are consistent in all three simulated configurations (Fig. 7). Here, the strain is investigated up to $1 \%$ because it is the typical level of detection interested in industrial structures, such as bridges and aircrafts. It should be noticed that each set of curves [blue (in online version), black, and red (in online version)] reflects the different $L c_{x}$ values only, while within each set, the difference between the solid and dash line curves reflect the strain simulations in which $L c_{x}$ is constant and the parameters $L p, W p, L s_{x}$, and $L$ loop $x$ are changed according to Table I. It can be observed that the resonator has the largest response for the changes in the set of parameters $\left\{L p, W p, L s_{x}, L \operatorname{loop}_{x}\right\}$ when $L c_{x}$ is around $9750 \mu \mathrm{m}$ and this response is altered not in a monotonic manner for other values of $L c_{x}$. This indicates that the two resonant frequencies are responsible by the two impedances coupled through the short stub ( $L s)$, one from the loop and another from the patch, generating a nonlinear response as the capacitance of the loop impedance changes.

In this design, the loop can be considered to be a modified stub that is loading on the patch. Based on the stub loading technique, tunable dual and triple resonant frequency patch antennas have been achieved [23], [24]. The design of the loop here is an effective method to maximize the capacitive change of the load (the loop) in response to the strain. Fig. 8 and 9 show the surface current distributions of the single loop design in Fig. 1 with

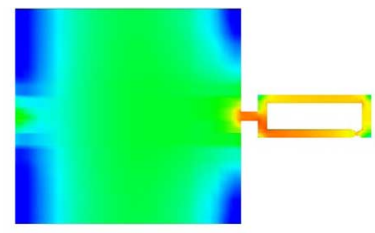

(a) $2.723 \mathrm{GHz}$

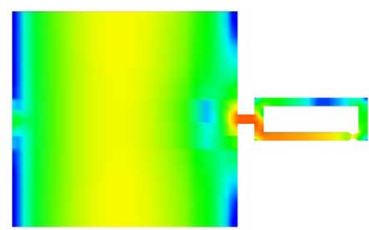

(a) $3.261 \mathrm{GHz}$
$-40$

Fig. 8. Surface current intensity of single loop model with $L c x$ of $9750 \mu \mathrm{m}$ for the nonstrain case at different resonant frequencies (solid black curve in Fig. 7).

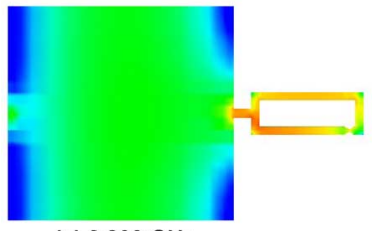

(a) $2.839 \mathrm{GHz}$

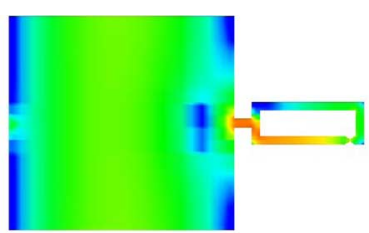

(a) $3.273 \mathrm{GHz}$
$-40$ $-20$ $-10$ Surface Current $(d \mathrm{~d})$

Fig. 9. Surface current intensity of single loop model with $L c x$ of $9750 \mu \mathrm{m}$ for the $1 \%$ strain case at different resonant frequencies (dashed black curve in Fig. 7).

parameter $L c_{x}$ of $9750 \mu \mathrm{m}$ in the cases of zero and $1 \%$ strain, respectively (the black curves in Fig. 7). We observe that the standing waves that are formed on the loop influence the fundamental mode of the patch, which causes the frequency shift. The patch loop structure can be considered to have two centers of resonant- or standing-wave regions, but the patch is still the effectively dominant radiator from which most radiation occurs. Since $|S 11|$ can be written as $\mid Z$ loop $-Z$ patch $|/| Z$ loop + $Z$ patch|, where $Z$ loop and $Z$ patch denotes the impedance of the loop and the patch, respectively, we observe that the rate of change of $|S 11|$ with respect to $\mid Z$ loop|, which contains a term of $L \mathrm{cap}_{x}$, is at least a second-order dependence. Therefore, such a rate of change would have at least a local maximum that is reflected in the nonmonotonic sensitivity of different $L c_{x}$ curves. To understand the nature of this relationship and the nonmonotonic response of different $L c_{x}$ curves, an equivalent-circuit model should be obtained for this open loop loaded patch structure. This will be the subject of future work, and therefore, this topic is not within the scope of this paper. On the other hand, $|S 11|$ levels in Fig. 7 appear to be high, indicating an impedance mismatch. Furthermore, we also observe from Fig. 7 that the reflection level is compromised by the sensor sensitivity. For example, the lower resonant frequency of the blue curves (in online version) $\left(L c_{x}=9500 \mu \mathrm{m}\right)$ has lower $|S 11|$ than that of the other curves, but the sensitivity in terms of frequency shift per $1 \%$ strain $(26 \mathrm{MHz})$ is lower than others, which are giving $66-$ and $125-\mathrm{MHz}$ shifts. To reduce the mismatch, additional matching elements can be added. However, to maintain the sensitivity at the same time would require further investigation and optimization of the design. At this stage of our work, we limit our scope to demonstrate the newly proposed principle of the cantilever integrated with a loop and a patch that can significantly improve the frequency shift with respect to the applied strain. 


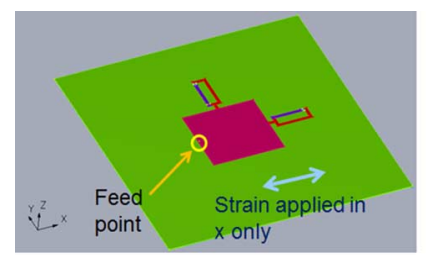

(a)

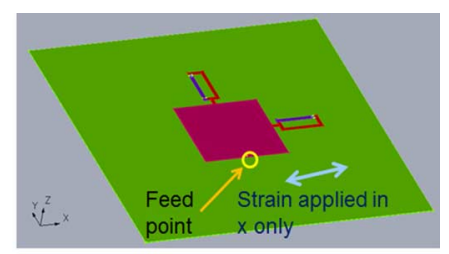

(b)
Fig. 10. Two configurations to simulate the two orthogonal modes of the rings loaded patch transducer (perpendicular excitation points). (a) $x$-polarization excitation. (b) $y$-polarization excitation.

\section{Study of the Second Operational Principle-Capability of Sensing in Multidimensional Independently}

Two different configurations of the structure in Fig. 2 are set up in order to investigate the responses of the transducer in different direction/polarization modes. The simulated models are shown in Fig. 10 where the strain is applied in the $x$-direction, while the feed point of each configuration is orthogonal to each other. The dimensions of the resonator under nonstrain and $1 \%$ strain conditions are shown in Table 1 corresponding to parameters indicated in Fig. 2. The simulation results of this study are shown in Fig. 11 with $L c_{x}=9750 \mu \mathrm{m}$, in which the solid lines represent the response of the $x$-polarization excitation and the dashed lines represent the response of the $y$-polarization excitation. Fig. 11 shows that around $2.8 \mathrm{GHz}$ when there is strain in the $x$-direction, a significant frequency shift is observed only for the $x$-polarization mode, while the response in the $y$-polarization mode stays relatively the same. Consequently, the strain in the $x$-direction does not influence the frequency response of the $y$-polarization excitation. Note that the resonant frequency is shifted by $110 \mathrm{MHz}(2.75-2.86 \mathrm{GHz})$, similar to the one observed for the simplified single loop structure of Section III-B (the black curve in Fig. 7), i.e., the sensitivity remains the same when another loop is added orthogonally. Therefore, the frequency shift observed in the $x$-polarization excitation can be attributed to the strain in the $x$-direction only. Since the dimensions $L p$ and $W p$ of the patch are slightly different, it can be identified that the $x$-polarization response has a $|S 11|$-peak around $3.3 \mathrm{GHz}$ and the $y$-polarization response has one around $3.4 \mathrm{GHz}$. These peaks have almost no shift in strain response since they are due to the patch; therefore, they can be used to correspond the frequency shift to the strain direction, i.e., the frequency shift around $2.8 \mathrm{GHz}$ can be identified to be caused by strain in the $x$-direction if the shift occurs in the curve containing the 3.3-GHz peak or $y$-direction if the shift occurs in the curve containing the $3.4-\mathrm{GHz}$ peak. As a result, the strain in $x$ and $y$ can be detected independently with this same transducer. Once the strain in the $x$ - and $y$-direction are obtained, the final strain in an arbitrary direction can be deduced by superposition of these two orthogonal strain responses because strain is essentially the shear force applied on the surface combining vectorially the two strain components (Fig. 5). Therefore, this mechanism allows indicating the strain in an arbitrary direction.

Note that the resonant frequency of the ring is not only highly sensitive to the capacitance area (Fig. 6), but also the gap between the cantilever and the other ring end ( $h$ cap in Fig. 4).

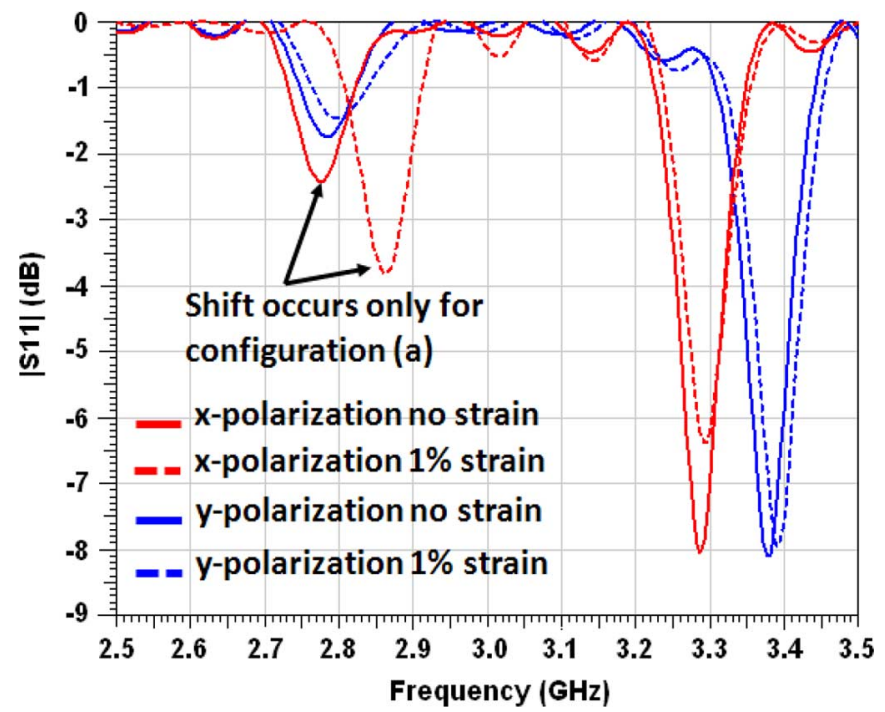

Fig. 11. Frequency response of the transducer in the two configurations when exciting in two different polarizations under nonstrain and $1 \%$ strain conditions.

Results from Fig. 7 show that mainly the capacitance area determines the sensitivity of the transducer in terms of frequency shift for a given strain. To show that the second principle still holds valid regardless of the gap capacitance area (Fig. 6 illustrated the overlap) or the gap size ( $h$ cap in Fig. 4), the width of the cantilever is changed to $800 \mu \mathrm{m}$ instead of $1000 \mu \mathrm{m}$, and $h$ cap is changed to $15 \mu \mathrm{m}$ instead of $10 \mu \mathrm{m}$ (compared to Table I). The simulation results are shown in Fig. 12 for the same strain conditions applied from Table I. Although the resonant frequency of the loop is relocated to around $3.6 \mathrm{GHz}$ instead of $2.8 \mathrm{GHz}$ in Fig. 7, the same behavior is observed, i.e., a significant shift of about $120 \mathrm{MHz}(3.67-3.55 \mathrm{GHz})$, i.e., $3.3 \%$, occurs only in $x$-polarization excitation. Observe that from Figs. 10 and 11 there exists a small shift of a about $20 \mathrm{MHz}$ in $y$-polarization excitation (3.61-3.63 GHz), i.e., $0.55 \%$. In principle, this small shift can be de-embedded in detection processing.

\section{Prototypes And Measurement Setup}

For the experimental verification of the above simulated trends, two prototypes of the proposed design in Fig. 2 were fabricated with two different feed positions exciting two orthogonal modes of the patch. The fabrication process was similar to the one described in [21] and [22]. The patch and the flat open ring portion of the circuit were fabricated on Kapton with $100-\mu \mathrm{m}$ thickness without the cantilever, while the cantilevers were fabricated separately from a $100-\mu \mathrm{m}$-thick aluminum sheet (Fig. 13). The cantilever was then manually assembled onto the patch circuit.

To prepare the patch circuit for assembly, the surface surrounding the tip of the ring (the portion on Kapton) was cleaned and a small amount of transparent adhesive glue spread as thin as possible was manually applied to create a $10-15-\mu \mathrm{m}$ insulating layer (Fig. 14); however, the thickness of this layer could not be controlled very accurately due to the manual process. After this glue layer is dry, the aluminum cantilever was soldered to the other end of the open loop (Fig. 14). To ensure the cantilever tip was in contact with the glue layer and fixed in the 


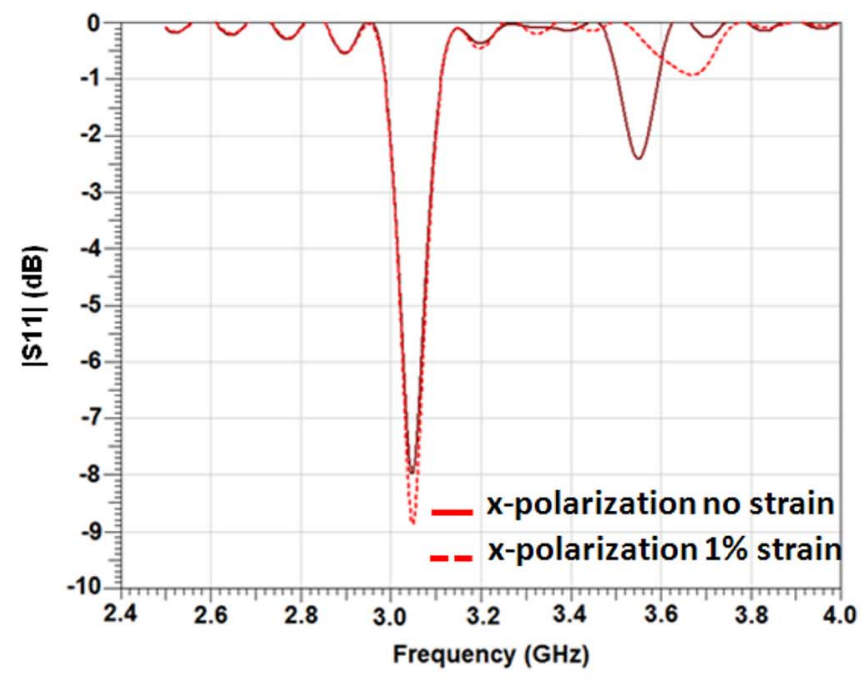

(a)

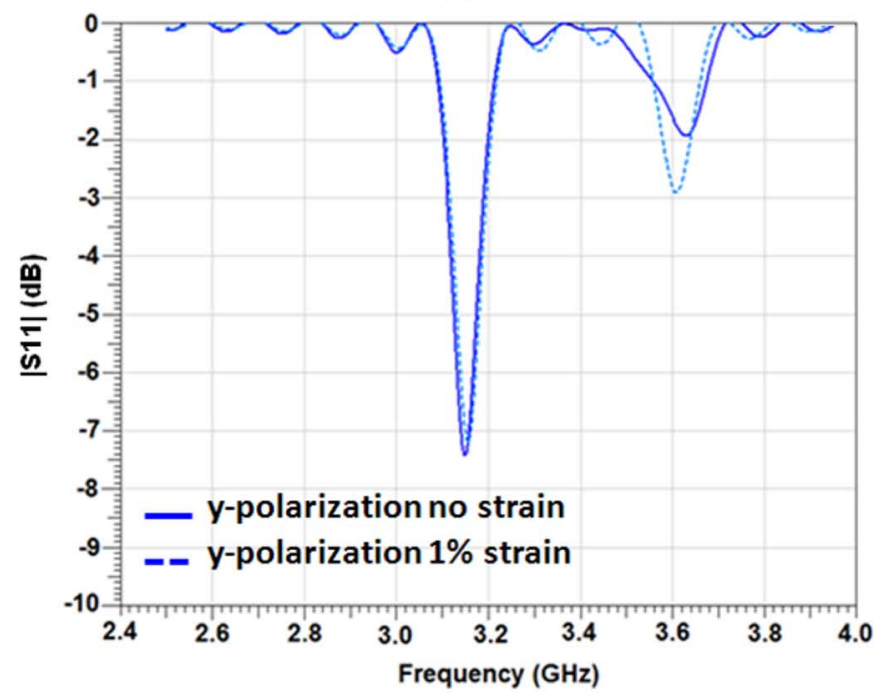

(b)

Fig. 12. Frequency responses of the 2-D strain transducer for $h$ cap of $15 \mu \mathrm{m}$ and cantilever width of $800 \mu \mathrm{m}$. (a) $x$-polarization excitation. (b) $y$-polarization excitation.

normal direction, but free to move in the elongation direction, Scotch tape was then used to cover over the surface (Fig. 14). In this configuration, as the open loop was stretched, the cantilever could still move freely as the soldered end of the cantilever pulls on it. The tape can also prevent the motion in a random arbitrary direction due to mechanical noise. The exerting force can move the cantilever only in the direction of the applied strain. The completed prototypes of the two different excited polarizations are shown in Fig. 15.

The measurement set up is shown in Fig. 16. In this figure, the strain patch sensor substrate has its top edge mounted to a fixed location on a ladder. The bottom edge of the Kapton substrate is attached to a variable load below. As the load increases when more weights are added, its gravity would pull on the substrate stretching its body in the vertical direction. As the substrate is stretched, the strain would be induced on the substrate surface. The sensor is connected to the vector network analyzer (VNA) with a coaxial connector shown near the top left in Fig. 16. The

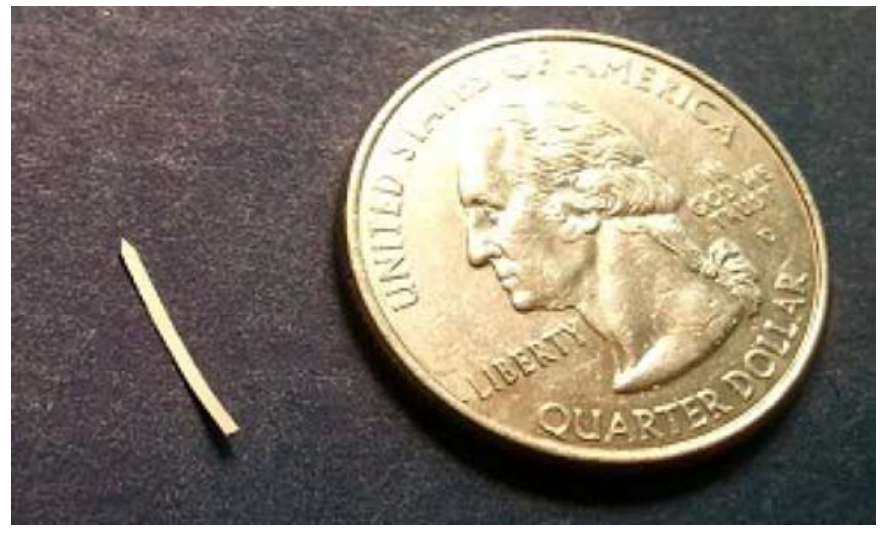

Fig. 13. Aluminum cantilever is fabricated separately from $100-\mu \mathrm{m}$ aluminum sheet.

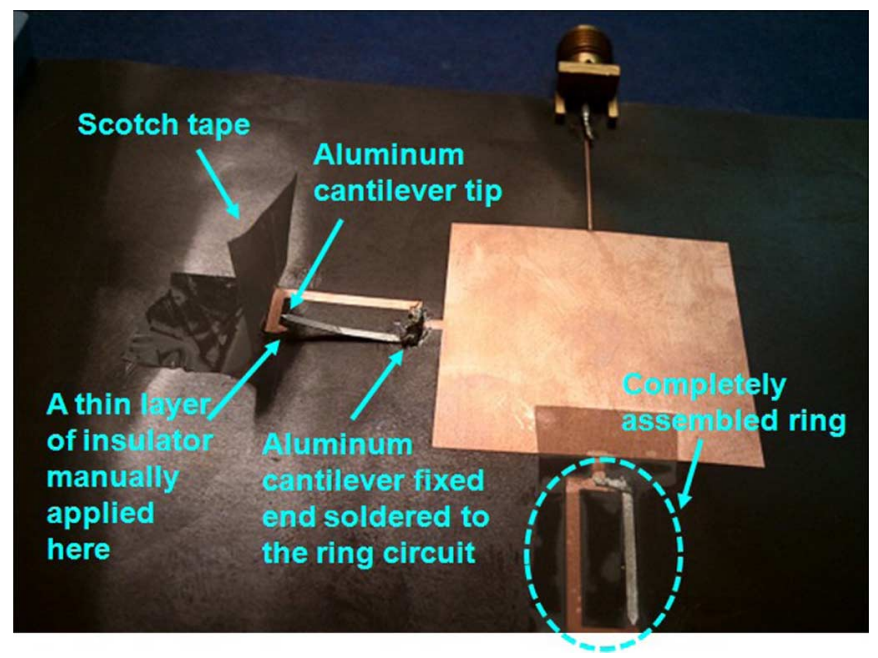

Fig. 14. Assembly of the aluminum cantilevers onto the patch circuit.

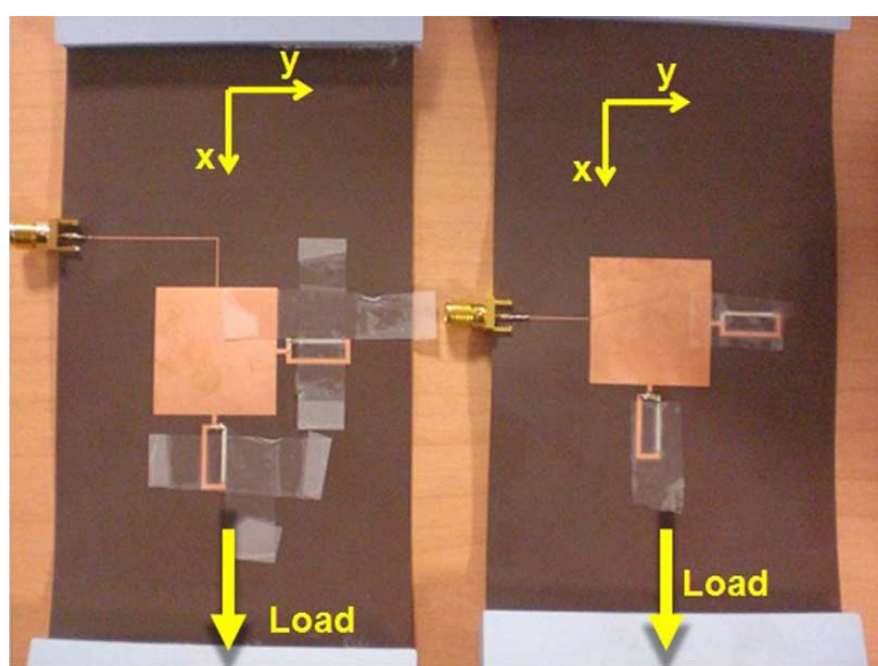

(a)

(b)

Fig. 15. Assembled prototypes. (a) $x$-polarization excitation. (b) $y$-polarization excitation.

VNA was calibrated with standard thru-reflect-line (TRL) procedure. Therefore, the frequency responses of the antenna can 


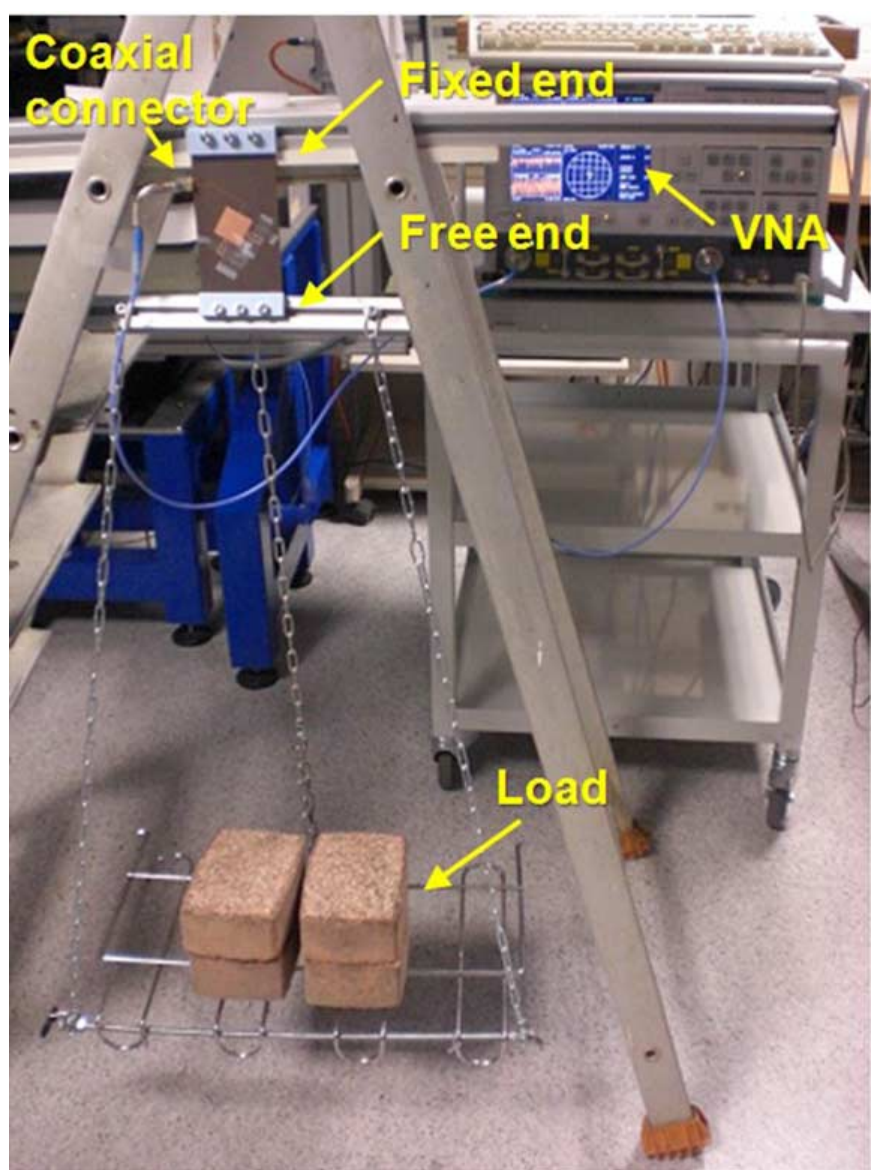

Fig. 16. Measurement setup.

be monitored in real time corresponding to the strain level induced by different loads. Due to the limited availability of loads, only irregular increments of load were implemented. Note that Fig. 16 illustrates the measurement setup only. The sample that appears in the figure is not a part of the measurements reported in Section. V. Instead, each sample presented in Fig. 15 was subjected to the measurement setup shown in Fig. 16. Due to a laborious manual process, it is difficult to accurately control the initial gap capacitance of the circuit since the $L$ cap can be easily varied between $750-1000 \mu \mathrm{m}$ (Fig. 6) and the gap size $h$ cap can be varied between $10-20 \mu \mathrm{m}$. Therefore, it is difficult to tune the circuit response to the operation point of the largest sensitivity response (Figs. 7 and 12). The strain resulting from this setup can be estimated according to (3) [17], where $P$ is the load, $E$ is the Young's modulus of Kapton (2.5 GPa), $t$ is the thickness of Kapton, and $W$ is the total width of the Kapton substrate, which is $150 \mathrm{~mm}$

$$
\varepsilon_{L}=\frac{P}{E \cdot t \cdot W} \cdot 100 \% .
$$

\section{Results AND Discussion}

The measurement results of the two prototypes in Fig. 15 are shown in Figs. 17-19 for different loads corresponding to different strain values. Fig. 17 shows the frequency responses of

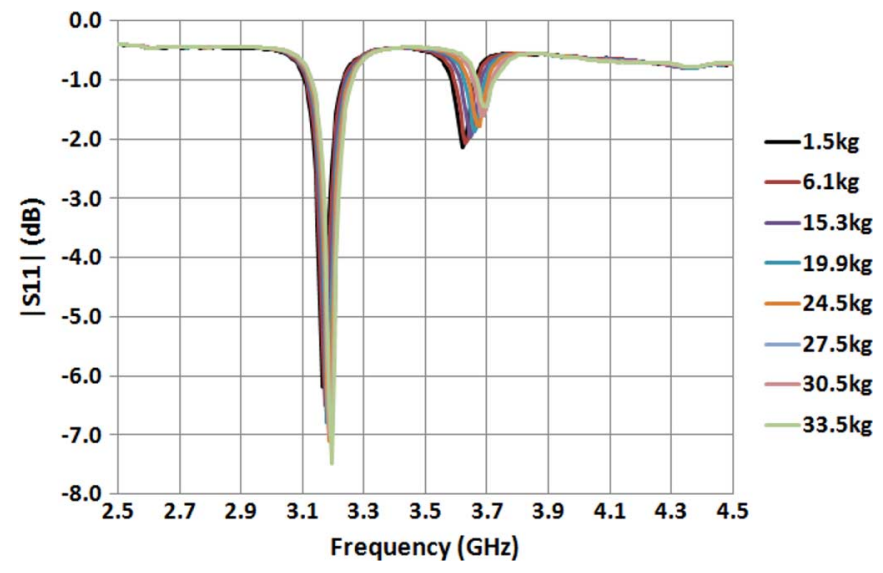

Fig. 17. Measured $|S 11|$ of the fabricated prototype with $x$-polarization excitation subjected to various attached weights.

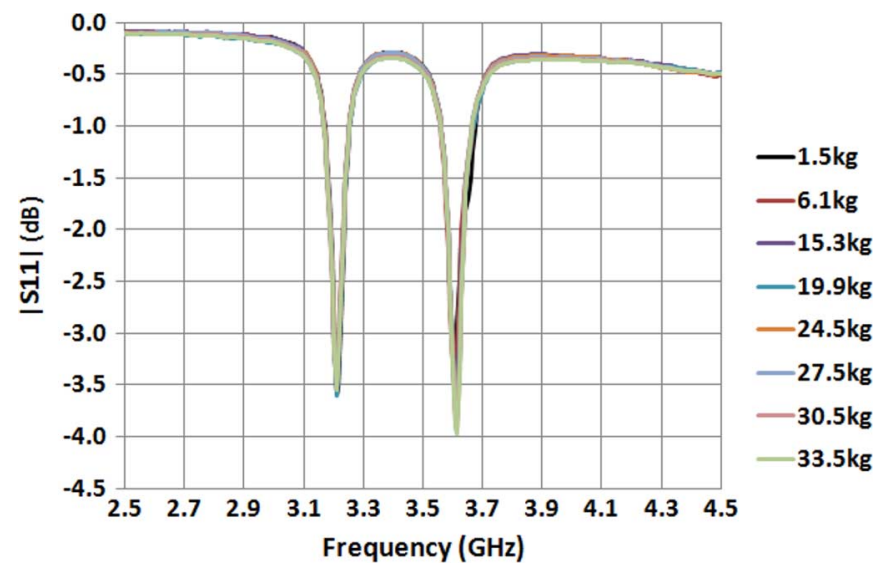

Fig. 18. Measured $|S 11|$ of the fabricated prototype with $y$-polarization excitation subjected to various attached weights.

the $x$-polarization excitation prototype under different loads in the $x$-direction, while Fig. 18 shows the frequency responses of the $y$-polarization excitation prototype under different loads also in the $x$-direction. Note that these frequency responses correspond to the simulations shown in Fig. 12 in which a thicker capacitance gap is investigated. This shows that the adhesive gel applied in this assemble is thicker than the one in the single loop prototype shown in the previous work [21], [22]. Nonetheless, it can be observed that the frequency shift only occurs in the $x$-polarization excitation prototype, thus validating the operational principles discussed in Section II.

A frequency shift of about $70 \mathrm{MHz}(3.62-3.69 \mathrm{GHz})$ can be observed in Fig. 19 where the magnitude of $S 11$ of the $x$-polarization excitation prototype is plotted around $3.6-3.7 \mathrm{GHz}$. This shift occurs for strain of $0.04 \%-0.88 \%$ calculated based on (3). The sensitivity in terms of frequency shift versus percentage of the applied strain is plotted in Fig. 20. The slope of the best fit curve gives a sensitivity of $2.35 \%$ frequency shift per $1 \%$ strain. This is more than two times higher than the sensitivity of the existing strain transducers of the same class. Based on the same design and operational principles, the highest sensitivity observed in simulations was shown to be about four times higher than existing transducers that produce about a $1 \%$ frequency shift per $1 \%$ strain (black curve in Fig. 7). Although the prototype is not 


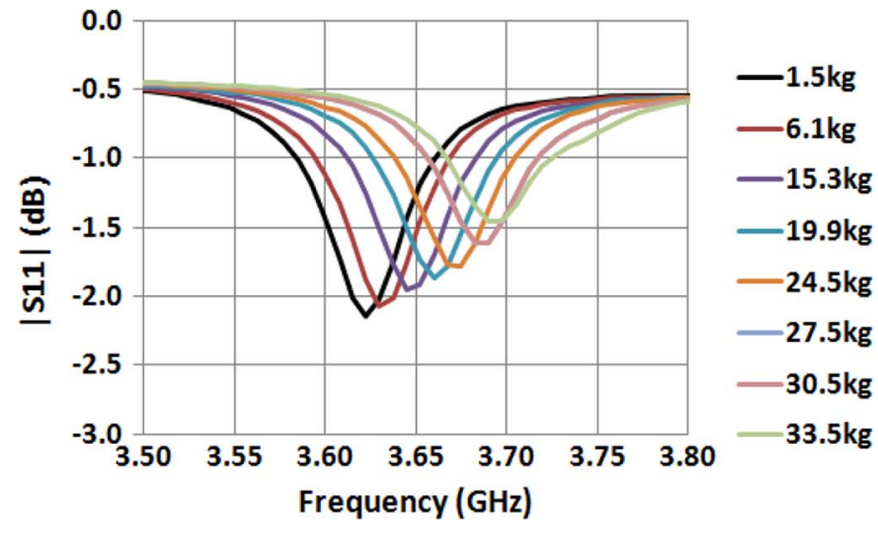

Fig. 19. Measured $|S 11| \mid$ around $3.6 \mathrm{GHz}$ of the fabricated prototype with $x$-polarization excitation subjected to various attached weights.

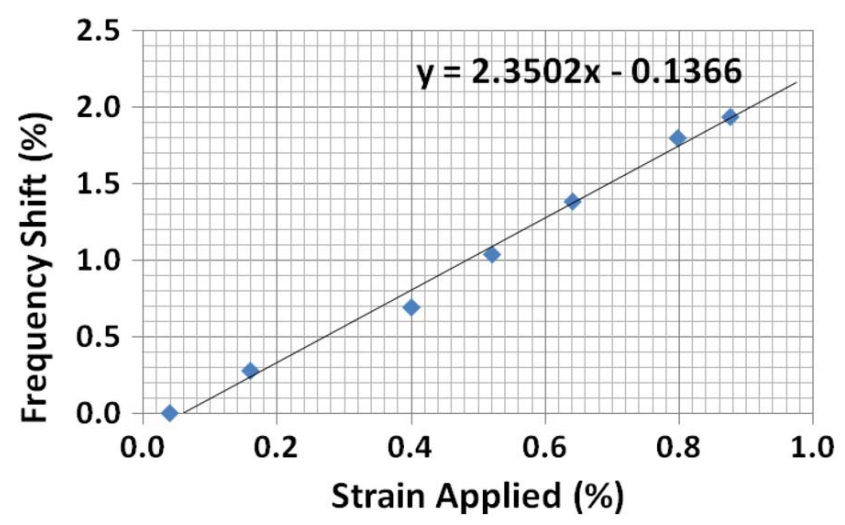

Fig. 20. Plot of $\%$ frequency shift versus $\%$ applied strain.

operating at the highest sensitive frequency point, these measurements have successfully validated the novel design and the new principles introduced in this work. The resolution of $|S 11|$ value, i.e., minimum detectable change, of the curves in Fig. 19 depends on the mechanism of the detection system which is discussed in Section VI. Fig. 19 also shows excellent linearity in the interested range of strain, i.e., up to $0.88 \%$ of strain. A typical strain level to be monitored in infrastructure and aerospace is up to $1 \%$ for structures such as buildings, bridges, and aircrafts.

\section{IMPLEMENTATION EXAMPLE OF THE WIRELESS PAssive Remote SENSING System FOR THE RF STRAIN TRANSDUCER}

The RF strain transducer can be implemented in completely passive sensing nodes for remote interrogation. The wireless measurement is based on the variation of the RCS level of loaded multiband scatterers read by an FMCW radar [25]. The scatterers here are the patches loaded with the open loop rings, i.e., the strain transducers. To illustrate the interrogation method that can exploit the frequency shift behavior of the proposed transducer, the RCS response of the sensor design presented in Fig. 1 is calculated and plotted based on the configuration presented in Fig. 21, in which a thin dipole antenna is terminated by the sensor (one-port patch structure) acting as a load. The well-known expression of the RCS level for this loading

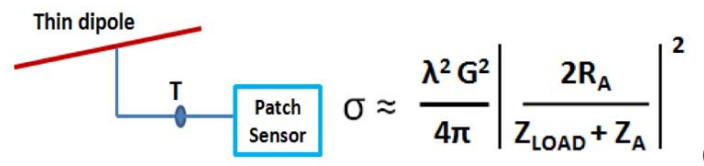

(4)

Fig. 21. Passive sensing configuration for RCS variation reading.

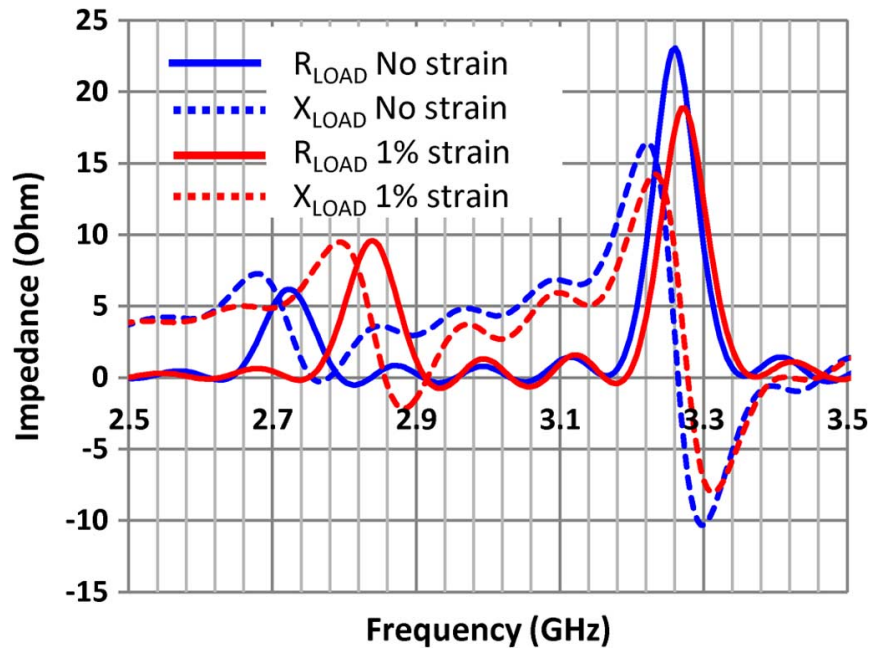

Fig. 22. Input impedance $Z_{\mathrm{LOAD}}$ of the patch-loop strain sensor when no strain (blue lines in online version) and $1 \%$ strain (red lines in online version) are applied.

configuration is shown in (4) [26]. Here, at a given operating frequency $\mathrm{f}(\lambda=c / f), G$ is the linear gain of the dipole antenna, $R_{A}$ is the real part of the complex input impedance $Z_{A}$ of the dipole, and $Z_{\mathrm{LOAD}}=R_{\mathrm{LOAD}}+j X_{\mathrm{LOAD}}$ is the complex input impedance of the patch-loop strain sensor. The simulation data obtained from Fig. 7 for $L c_{x}$ of $9759 \mu \mathrm{m}$ (the solid and dash black curves) gives the input impedance $Z_{\mathrm{LOAD}}$ of the sensor corresponding to no strain and $1 \%$ strain applied (Fig. 22). This input impedance is utilized in the calculations of (4) together with the input impedance of the thin dipole to derive the RCS responses (Fig. 23). The dipole antenna is designed to resonate at $2.722 \mathrm{GHz}$ (52 $\mathrm{mm}$ in length), which is the resonant peak of the lower resonant frequency observed in the solid black curve of Fig. 7. The response shows an RCS variation of about $0.73 \mathrm{~dB}$ corresponding to the strain applied.

In order to increase the RCS variation observed in Fig. 23, an impedance transformer is designed and presented in Fig. 24 with the assistance of the package Advanced Design System (ADS) by Agilent Technologies. The input impedance $Z_{\mathrm{IN}}=$ $R_{\mathrm{IN}}+j X_{\mathrm{IN}}$ of the patch-loop strain sensor after applying the impedance transformer is shown in Fig. 25. At the system level, the transformer is inserted at point $T$ in Fig. 21, where port 1 looks into the thin dipole, while port 2 looks into the patch-loop sensor feed point. Therefore, we observe that the new input impedance $Z_{\mathrm{IN}}$ of the load (the patch-loop sensor) is scaled by a complex factor due to the parallel impedance $Z_{\mathrm{CAP} A}=$ $1 / j \omega C$, where $\omega=2 \pi f$ and $C=113 \mathrm{fF}$, and the scaling factor itself is also proportional to the variations of the load nonlinearly, as illustrated in (5). The resulting RCS of the thin dipole is then recalculated and shown in Fig. 26 yielding an RCS variation of about $3.8 \mathrm{~dB}$, a detectable level. Consequently, the RCS 


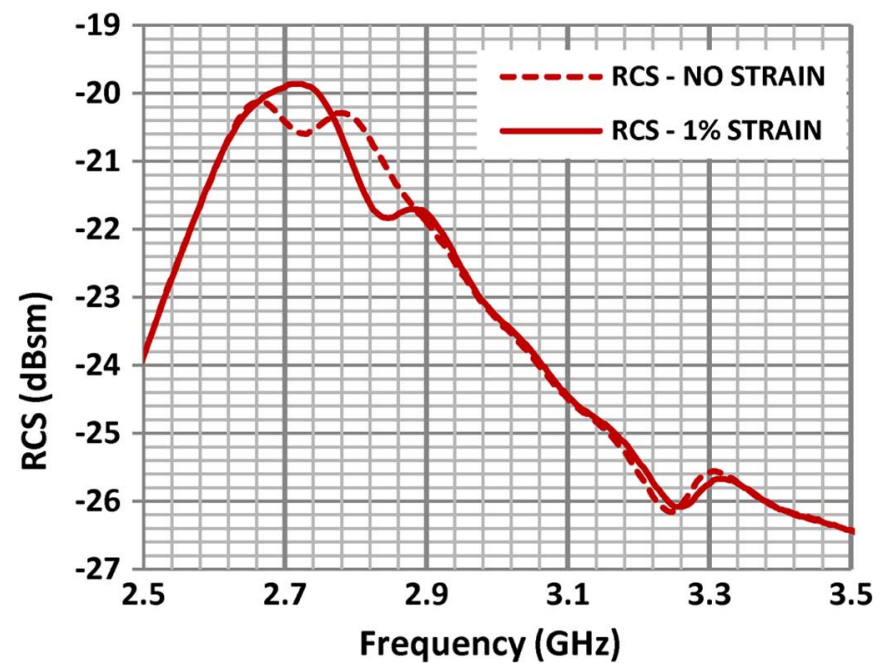

Fig. 23. RCS responses due to the loading effect of the strain transducer (without impedance transformer).

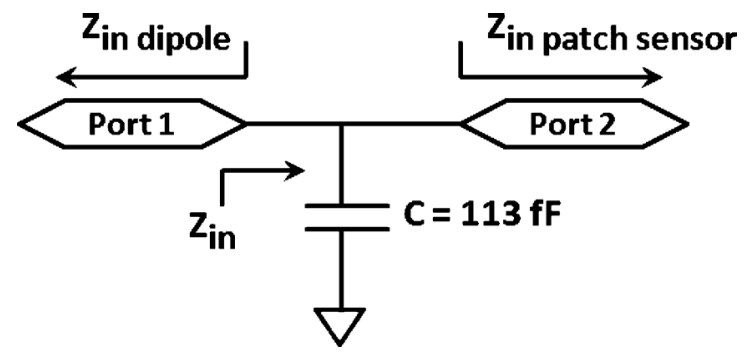

Fig. 24. Single parallel capacitance $C$ connected at the input port of the patchloop strain sensor allows reaching detectable RCS variation of around $3.8 \mathrm{~dB}$ (see Fig. 27).

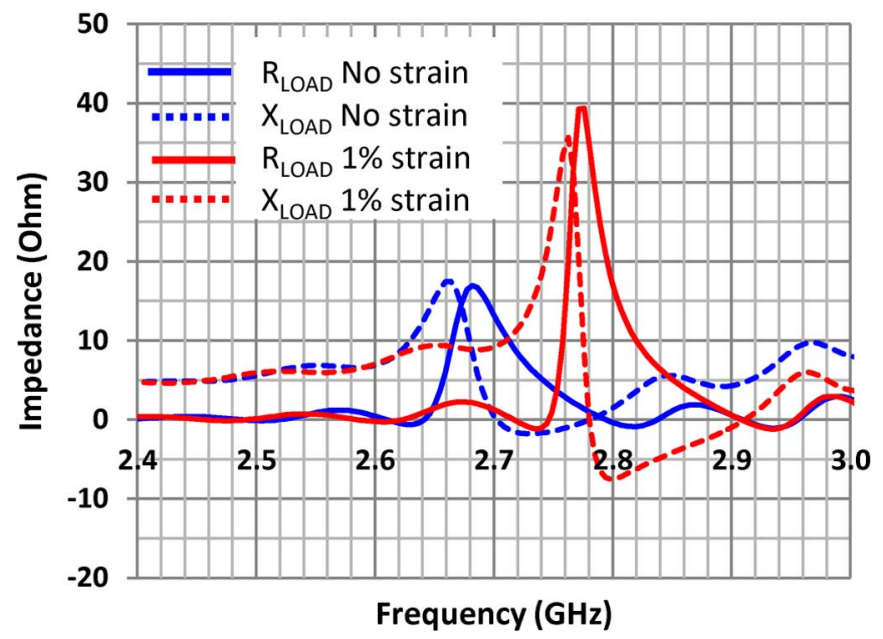

Fig. 25. Input impedance $Z_{\mathrm{IN}}=R_{\mathrm{IN}}+j X_{\mathrm{IN}}$ of the patch-loop strain sensor after applying the impedance transformer when no strain (blue lines in online version) and $1 \%$ strain (red lines in online version) are applied.

calculations illustrate the feasibility of such interrogation architecture and can be improved with further development. Such a fluctuation of the RCS level can be potentially further applied to a communication network illustrated in Fig. 27 utilizing delay lines

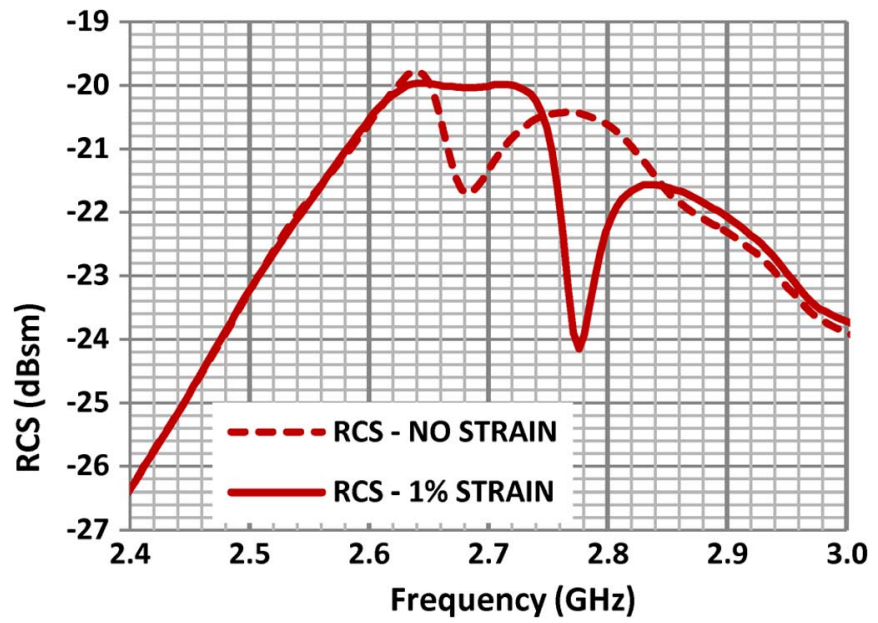

Fig. 26. RCS responses due to the loading effect of the strain transducer after applying the impedance transformer to the patch-loop sensor.

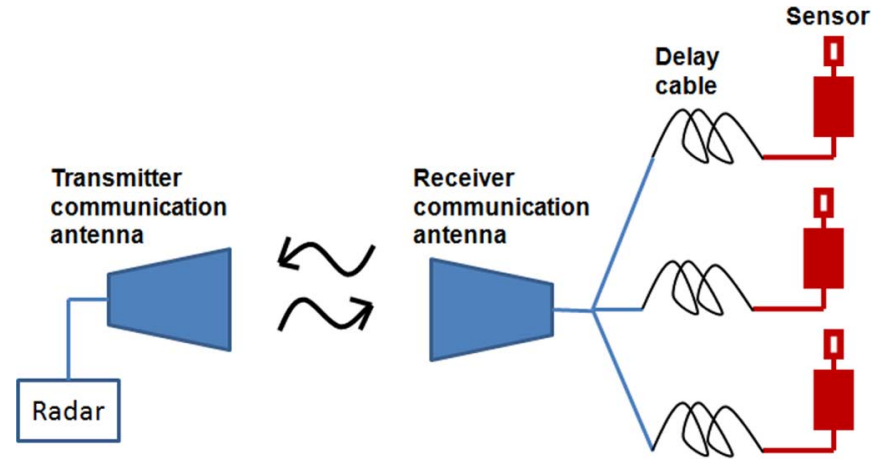

Fig. 27. RCS measurement system for remote sensing and identification based on FMCW radar reader.

$$
\begin{aligned}
Z_{\mathrm{IN}} & =\left(Z_{\mathrm{CAP} A} / / Z_{\mathrm{LOAD}}\right) \\
& =\frac{R_{\mathrm{LOAD}}+j Z_{\mathrm{LOAD}}}{1-\omega C X_{\mathrm{LOAD}}+j \omega C R_{\mathrm{LOAD}}} .
\end{aligned}
$$

In a sensing system, sensor nodes should not only provide information about physical conditions of the surrounding environment, but also provide their identification in the network of multiple sensor nodes. Therefore, based on the technique recently reported in [16], [25], [27], and [28], the same concept is suggested for the implementation of the strain sensor to allow for an efficient communication and discrimination between networks of many passive sensor nodes with a central monitoring station.

The "reader" loaded horn antenna is usually located several meters away from the terminal of the radar system. Delay cables are connected to different feeding points on the patches to terminate them with either match load, open, or short. In this technique of remote identification and data acquisition, the radar emits RF signals, which would travel through the load system, reflect at the terminal, and re-emit from the sensors with some delays due to the RF cable. The radar ("reader") receives a modified response from the sensors while signals scattered from the rest of the environment remain unmodified. Based on the total 
travel distance of the signals, each object gives a different beat frequency with a different RCS level. The receiver at the radar may have polarization filters to differentiate signals from the two orthogonal modes of the patches. Therefore, it is capable of reading the strain change indicated by the frequency shifts based on the RCS fluctuations. With different lengths of the transmission lines connecting the sensor nodes (Fig. 27), the positions of different sensor nodes in this passive sensing network can be identified (indicated by beat frequencies) along with their sensing information (indicated by RCS levels). It is also possible to utilize slightly different operating frequencies (the orthogonal modes of the patches) for a group of sensors in a unit cell when implementing on large surface for sensing with high resolution. In utilizing this transducer in a passive sensor network similar to the one proposed here, the minimum detectable frequency shift is essentially limited by the radar noise level that allows it to detect a minimum fluctuation in the RCS level, which, in turn, depends on $|S 11|$ at a given frequency. This resolution can be improved further with impedance transformers as shown and/or by scaling the design to the millimeter-wave frequency range in which the noise level, and thus, the minimum detectable level of the FMCW radar, can be smaller. In principle, the final sensitivity resolution also depends on the surface deformation of the substrate, i.e., Kapton, for a given strain applied. However, the elasticity of Kapton is much larger than the surface of the structures monitoring materials such as steel and concrete; consequently, the sensitivity of the transducer is only limited by the frequency shift for a given deformation.

\section{CONCLUSION}

A novel design of a highly sensitive wireless and passive 2-D strain RF transducer has been introduced. The newly proposed principles of operation have been illustrated and successfully validated through experiments. These ideas enable a new method of engineered sensitivity for utilizing this type of strain sensing process, which previously was limited to the linear scale of the changes in the lateral dimensions of the structures' surfaces. By utilizing the patch resonators, the sensitivity of the proposed design is independent from the material of the applied surface, yet with novel rings loaded to the patch, a high sensitivity is achieved. The prototypes give a sensitivity of $2.35 \%$ frequency shift per $1 \%$ strain, which is more than twice the sensitivity of existing sensors of the same class. In theory, the newly introduced principles allow a sensitivity of up to four times higher than previous solutions. Due to limited fabrication capability and rapid assembling, the prototype cannot be built to function at the best operating frequency point; therefore, the sensitivity achieved in the current prototype is limited. To the best knowledge of the authors, the design is also the first of its kind to be capable of sensing strain in arbitrary directions, although results were not directly measured, but directly deduced from the decomposition principle of shear forces. As an example, for a specific remote and passive sensing implementation, the sensor can be interrogated by a radar to monitor its RCS level change as an indicator of strain. A well detectable RCS level change of at least $3.8 \mathrm{~dB}$ achieved with the impedance transformer of a single parallel capacitor is shown in calculations based on the proposed interrogation method. This work illustrates feasibility for remote measurements of a strain vector field of a surface. With the well advanced microelectromechanical systems (MEMS) technology and the simplicity of the design, a low-cost manufacturing approach of this design can be readily obtained, which is the next step of this work. Apart from the RCS detection architecture in which smaller targets become harder to show its RCS fluctuations, the sensor and its working principles can be easily scaled to operate at millimeter-wave frequencies for higher resolution of strain sensing or adapted to biomedical applications at lower frequencies. The challenges in scaling to millimeter-wave bands are mostly associated with the fabrication of the cantilevers and stabilizing their positions in operation. Fortunately, the same electromagnetic principle of this transducer still holds valid so the structure only needs to be scaled according to the relationship between operational frequencies and physical dimensions. Although the RCS reading for the sensor at millimeter-wave bands can be challenging, the operation of the strain transducer has been demonstrated successfully in theory and experiments, and the interrogation method has also been verified theoretically.

\section{ACKNOWLEDGMENT}

The authors are thankful to the generous assistance of T. Idda, CNRS-LAAS, Toulouse, France, with measurements, as well as the help of X. Dollat, CNRS-LAAS, with fabrication.

This work was performed at CNRS-LAAS.

\section{REFERENCES}

[1] D. N. Farhey, "Long-term performance monitoring of the tech 21 allcomposite bridge," J. Compos. Construct., vol. 9, no. 3, pp. 255-262, 2005.

[2] P. C. Chang, A. Flataus, and S. C. Liu, "Review paper: Health monitoring of civil infrastructure," Struct. Health Monitor., vol. 2, no. 3, pp. 257-267, 2003

[3] B. Rao, "The need for condition monitoring and maintenance management in industries," in Handbook of Condition Monitoring. Amsterdam, The Netherlands: Elsevier, 1996, pp. 1-36.

[4] F. Gattiker, F. Umbrecht, D. Müller, and J. Neuenschwander, "Novel ultrasound read-out for a wireless implantable passive strain sensor (WIPSS)," Sens. Actuators A, Phys., pp. 291-298, 2008.

[5] K. Stoffel, K. Klaue, and S. M. Perren, "Functional load of plates in fracture fixation in vivo and its correlate in bone healing," Injury, vol. 31, no. 2, pp. 37-86, May 2000.

[6] Y. Liu, A. Lacher, G. Wang, A. Purekar, and M. Yu, "Wireless fiber optic sensor system for strain and pressure measurements on a rotor blade," Proc. SPIE, vol. 12, pp. 67700Y-67700Y, 2007.

[7] W. Staszewski, "Monitoring on-line integrated technologies for operational reliability-MONITOR," Air and Space Eur., vol. 2, no. 4, pp. 67-72, 2000.

[8] K. Sun, "Design and characterization of passive wireless strain sensor," Masters thesis, Univ. Puerto Rico, Mayaguez, PR, USA, 2006.

[9] J. Wu, S. Yuan, S. Ji, G. Zhou, Y. Wang, and Z. Wang, "Multi-agent system design and evaluation for collaborative wireless sensor network in large structure health monitoring," Expert Syst. Appl., vol. 37, no. 3, pp. 2028-2036, 2009.

[10] F. Ansari, "Fiber optic health monitoring of civil structures using long gage and acoustic sensors," Smart Mater. Struct., vol. 14, no. 3, pp. S1-S7, 2005.

[11] B. F. Spencer, Jr., M. E. Ruiz-Sandoval, and N. Kurata, "Smart sensing technology: opportunities and challenges," Struct. Control Health Monitor., vol. 5, no. 1, pp. 29-43, 2006.

[12] D. N. Farhey, "Integrated virtual instrumentation and wireless monitoring for infrastructure diagnostics," Struct. Health Monitor., vol. 5, no. 1, pp. 29-43, 2006. 
[13] R. Chacon, F. Guzman, E. Mirambell, E. Real, and E. Onate, "Wireless sensor networks for strain monitoring during steel bridges launching," Strut. Health Monitor., vol. 8, no. 3, pp. 195-205, 2009.

[14] J. Chuang, D. J. Thomson, and G. E. Bridges, "Embeddable wireless strain sensor based on resonant RF cavities," Rev. Sci. Instrum., vol. 76, no. 9, 2005, Art. ID 094703.

[15] M. Jatlaoui, P. Pons, and H. Aubert, "Pressure Micro-sensor based on radio frequency transducer," in IEEE MTT-S Int. Microw. Symp. Dig., Jun. 2008, pp. 1203-1206.

[16] T. Thai, F. Chebila, M. Jatlaoui, P. Pons, H. Aubert, G. DeJean, M. Tentzeris, and R. Plana, "Design and development of a millimetre-wave novel passive ultrasensitive temperature transducer for remote sensing and identification," in Eur. Microw. Conf., Sep. 2010, pp. $45-48$.

[17] U. Tara, H. Huang, R. Carter, and J. Chiao, "Exploiting a patch antenna for strain measurements," Meas. Sci. Technol., vol. 20, no. 1, 2009, Art. ID 015201.

[18] Z. Qian, Q. Tang, J. Li, H. Zhao, and W. Zhang, "Analysis and design of a strain sensor based on a microstrip patch antenna," in Int. Microw. Millim. Wave Technol. Conf., 2012, vol. 5, pp. 1-3.

[19] A. Daliri, A. Galehdar, S. John, W. Rowe, and K. Ghorbani, "Circular microstrip patch antenna strain sensor for wireless structural health monitoring," in Proc. World Congr. England, London, U.K., Jun.-Jul. 2010, vol. 2, pp. 1173-1178.

[20] R. Melik, E. Unal, N. K. Perkgoz, C. Puttlitz, and H. V. Demir, "Metamaterial-based wireless strain sensors," Appl. Phys. Lett., vol. 95, no. 1, 2009, Art. ID 011106.

[21] T. Thai, H. Aubert, P. Pons, M. Tentzeris, and R. Plana, "Design of a highly sensitive wireless passive RF strain transducer," in IEEE MTT-S Int. Microw. Symp. Dig., Baltimore, MD, USA, Jun. 2011.

[22] T. T. Thai, H. Aubert, P. Pons, R. Plana, G. R. DeJean, and M. M. Tentzeris, "A newly developed radio frequency wireless passive highly sensitive strain transducer," in IEEE Sensors Conf., Limerick, Ireland, Oct. 2011, pp. 211-214.

[23] A. E. Daniel and G. Kumar, "Tunable dual and triple frequency stub loaded rectangular microstrip antennas," in IEEE AP-S Int. Symp., 1995, vol. 4, pp. 2140-2143.

[24] E. Erdil et al., "Frequency tunable microstrip patch antenna using RF MEMS technology," IEEE Trans. Antennas Propag., vol. 55, no. 4, pp. 1193-1196, Apr. 2007.

[25] F. Chebila, M. Jatlaoui, P. Pons, and H. Aubert, "Pressure measurement from the radar interrogation of passive sensors," in IEEE Int. Antennas Propag. Symp., Toronto, ON, Canada, Jul. 2010, pp. 1-4.

[26] C. Balanis, Antenna Theory Analysis and Design, 2nd ed. New York, NY, USA: Wiley, 1997, pp. 95-96.

[27] M. M. Jatlaoui, F. Chebila, P. Pons, and H. Aubert, "New micro-sensors identification techniques based on reconfigurable multi-band scatterers," in Asia-Pacific Microw. Conf., Singapore, Dec. 7-10, 2009, pp. 968-971.

[28] M. M. Jatlaoui, F. Chebila, P. Pons, and H. Aubert, "Wireless interrogation techniques for a passive pressure micro-sensor using an EM transducer," in Eur. Microw. Week, Rome, Italy, Sep.-Oct. 2009, pp. 053-056.

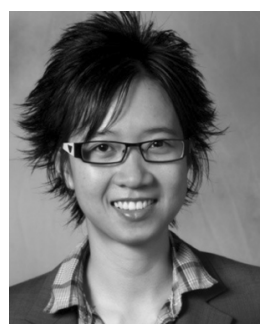

Trang T. Thai (S'05) received the B.Sc. degree in electrical engineering (with Honors) and B.Sc. degree in physics (with Honors) from the Georgia Institute of Technology, Atlanta, GA, USA, in 2008, and is currently working toward the Ph.D. degree in electrical engineering at the Georgia Institute of Technology. Her thesis concerned soft hard surface for high gain compact antennas.

Since 2009, she has been a Visiting Researcher (on a scholarship) with the Laboratoire d'Analyse et d'Architecture des Systèmes (LAAS), National Center for Scientific Research (LAAS-CNRS), Toulouse, France. From 2010 to 2012, she was with Microsoft Research, as a Research Intern for three summers. While with Microsoft Research, she developed a new proximity sensor for gesture recognition, on-body communication channels, and other wearable sensing platforms. She has authored or coauthored over 25 papers in refereed journals and conference proceedings. She contributed to a book chapter in Passive RF Component Technology Materials, Techniques, and Applications (Artech House, 2012). She holds three patents, two with Microsoft and one with LAAS-CNRS. Her research is focused on RF sensing with a multidisciplinary approach. Her past and current research includes designs and developments of the RF transducers for pressure, temperature, and strain sensing, all of which pioneer a new class of transducers that are based on RF principles enabling passive and remote sensing. Her other research includes gas sensing based on nanomaterials and surface plasmon RF sensing applications.

Ms. Trang was the recipient of the IEEE Microwave Theory and Techniques Society (IEEE MTT-S) Undergraduate/Pre-Graduate Scholarship Award in 2007. She was also a recipient of the prestigious Microsoft Research Ph.D. Fellowship in 2011.

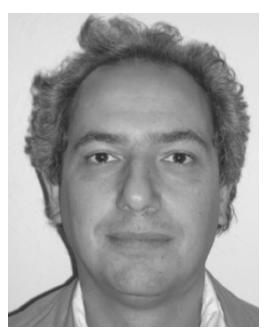

Hervé Aubert (M'94-SM'99) was born in Toulouse, France, in July 1966. He received the Eng. Dipl. and Ph.D. degree (with high honors) in electrical engineering from the Institut National Polytechnique (INPT), Toulouse, France, in 1989 and 1993, respectively.

Since February 2001, he has been a Professor with INPT. In February 2006, he joined the Laboratoire d'Analyse et d'Architecture des Systèmes (LAAS), National Center for Scientific Research (CNRS), Toulouse, France. From April 1997 to March 1998, he was a Visiting Associate Professor with the School of Engineering and Applied Science, University of Pennsylvania, Philadelphia, PA, USA. From July 2001 to January 2005, he was co-chairman of the Electronics Laboratory, INPT, and from July 2002 to September 2005, the Head of the Electromagnetics Research Group. From September 2004 to September 2011, he was the Director of the Research Master Program in Microwaves, Electromagnetism and Optoelectronics, Toulouse, France. Since January 2012, he has been the co-chairman of the Micro- and Nano-Systems for Wireless Communications Research Group, LAAS-CNRS. He contributed to the books Fractals: Theory and Applications in Engineering (Springer, 1999), Micromachined Microwave Devices and Circuits (Romanian Academy, 2002) and New Trends and Concepts in Microwave Theory and Techniques (Res. Signpost, 2003). He has authored or coauthored one book, two book chapters, 64 papers in refereed journals, and over 160 communications in International Symposium Proceedings. He holds four international patents in the area of antennas. $\mathrm{He}$ has performed research on integral-equation and variational methods applied to electromagnetic wave propagation and scattering. His current research activities involve the electromagnetic modeling of complex (multiscale) structures.

Dr. Aubert has been an expert for the French Research Agency (ANR) since 2009 and an expert for the European Commission since 2012. He is the secretary of IEEE Antennas and Propagation French Chapter. He was the vice-chairman of this chapter from 2004 to 2009 and secretary from 2001 to 2004.

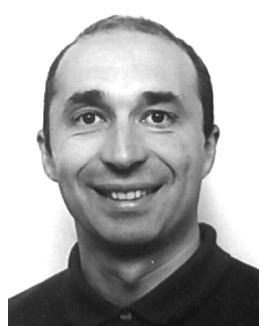

Patrick Pons received the Ph.D. degree in electronics from Toulouse University, Toulouse, France, in 1990 .

Since 1991, he has been a Researcher with the Laboratoire d'Analyse et d'Architecture des Systèmes (LAAS), National Center for Scientific Research (CNRS), Toulouse, France. His research interests are focused on microtechnology and microsensors. In 1995, he began laboratory study on microtechnology for microwave applications. He currently manages the development of this technology for high-frequency microsystems and also develops pressure sensors for specific applications. In 2005, he began research in a new field coupling sensors and RF for the development of passive wireless sensors.

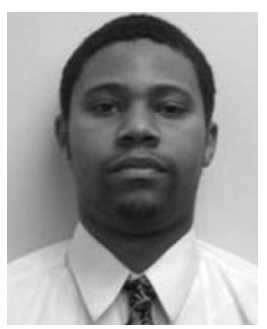

Gerald R. DeJean (S'03-M'06) received the B.S. degree in electrical and computer engineering from Michigan State University, East Lansing, MI, USA, in 2000, and the M.S. and Ph.D. degrees in electrical and computer engineering from the Georgia Institute of Technology, Atlanta, GA, USA, in 2005 and 2007, respectively.

He is currently with Microsoft Research, Redmond, WA, as a Researcher involved in the field of RF and antenna design. In November 2008, he became an Adjunct Assistant Professor with the Georgia Institute of Technology. He has authored or coauthored over 60 papers in refereed journals and conference proceedings. His research interests include antenna design, $\mathrm{RF} /$ microwave design and characterization, and 3-D 
system-on-package (SOP) integration of embedded functions that focuses largely on modern commercial RF systems such as cellular phones for PCS applications, Bluetooth, and 2.4-GHz industrial-scientific-medical (ISM) applications, RF identifications (RFIDs), wireless local area networks (WLANs) (802.11 a,b,g), LMDS, and millimeter-wave applications at $60 \mathrm{GHz}$. He has dedicated his research to making the antenna more compact and integrable with multilayer packages such as low-temperature co-fired ceramic (LTCC) liquid crystal polymer (LCP), and multilayer organic (MLO) while maintaining the full functionality of the device for wideband and/or multiband applications. $\mathrm{He}$ is also interested in equivalent circuit modeling techniques to assist in the design and optimization of compact wireless devices. Furthermore, he conducts research in remote sensing technologies in the form of pressure, temperature, and user proximity. He is currently involved with the implementation of on-body communications for health monitoring and ubiquitously secure data transfer.

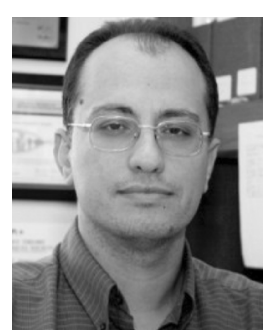

Manos M. Tentzeris (S'89-M'92-SM'03-F'10) received the Diploma degree in electrical and computer engineering from the National Technical University of Athens (magna cum laude) from the National Technical University, Athens, Greece, and the M.S. and Ph.D. degrees in electrical engineering and computer science from The University of Michigan at Ann Arbor, Ann Arbor, MI, USA.

$\mathrm{He}$ is currently a Professor with School of Electrical and Computer Engineering, Georgia Institute of Technology, Atlanta, GA, USA. He has authored or coauthored over 420 papers in refereed journals and conference proceedings, five books, and 19 book chapters. He is an Associate Editor for the International Journal on Antennas and Propagation. He has helped develop academic programs in highly integrated/multilayer packaging for RF and wireless applications using ceramic and organic flexible materials, paper-based RFIDs and sensors, biosensors, wearable electronics, inkjet-printed electronics, "green" electronics and power scavenging, nanotechnology applications in RF, microwave MEMs, system-on-package (SOP) integrated (ultra-wideband (UWB), multiband, millimeter wave, conformal) antennas, and heads the ATHENA Research Group (20 researchers). He is currently the Head of the Electromagnetics Technical Interest Group, School of Electrical and Computer Engineering, Georgia Institute of Technology, and has served as the Georgia Electronic Design Center Associate Director for RFID/Sensors research from 2006 to 2010 and as the Georgia Institute of Technology National Science Foundation (NSF) Packaging Research Center Associate Director for RF Research and the RF Alliance Leader from 2003 to 2006. For the summer of 2002, he was a Visiting Professor with the Technical University of Munich, Munich, Germany. For the summer of 2009, he was a Visiting Professor with GTRI-Ireland, Athlone, Ireland, For the summer of 2010, he was a Visiting Professor with Laboratoire d'Analyse et d'Architecture des Systèmes (LAAS), National Center for Scientific Research (CNRS), Toulouse, France. He has given over 100 invited talks to various universities and companies worldwide.

Dr. Tentzeris is a member of URSI, Commission D and the MTT-15 Committee. He is an Associate Member of the European Microwave Association (EuMA). He is a Fellow of the Electromagnetic Academy. He is a member of the Technical Chamber of Greece. From 2010 to 2012, he was an IEEE Microwave Theory and Techniques Society (IEEE MTT-S) Distinguished Microwave Lecturer. He was the Technical Program Committee chair for the 2008 IEEE MTT-S International Microwave Symposium (IMS) and the chair of the 2005 IEEE CEM-TD Workshop. He is the vice-chair of the RF Technical Committee (TC16), IEEE CPMT Society. He is the founder and chair of the RFID Technical Committee (TC24) of the IEEE MTT-S and the secretary/treasurer of the IEEE C-RFID. He is an associate editor for the IEEE TRANSACTIONS ON Microwave THEORY AND TECHNIQUES and the IEEE TRANSACTIONS ON ADVANCED PACKAGING. He was the recipient/corecipient of the 2012 FiDiPro Award in Finland, the 2010 IEEE Antennas and Propaga- tion Society Piergiorgio L. E. Uslenghi Letters Prize Paper Award, the 2011 International Workshop on Structural Health Monitoring Best Student Paper Award, the 2010 Georgia Institute of Technology Senior Faculty Outstanding Undergraduate Research Mentor Award, the 2009 IEEE TRANSACTIONS ON Components and Packaging Technologies Best Paper Award, the 2009 E. T. S. Walton Award from the Irish Science Foundation, the 2007 IEEE AP-S Symposium Best Student Paper Award, the 2007 IEEE MTT-S IMS Third Best Student Paper Award, the 2007 ISAP 2007 Poster Presentation Award, the 2006 IEEE MTT-S Outstanding Young Engineer Award, the 2006 Asia-Pacific Microwave Conference Award, the 2004 IEEE TRANSACTIONS ON AdVANCED PACKAGING Commendable Paper Award, the 2003 NASA Godfrey "Art" Anzic Collaborative Distinguished Publication Award, the 2003 IBC International Educator of the Year Award, the 2003 IEEE CPMT Outstanding Young Engineer Award, the 2002 International Conference on Microwave and Millimeter-Wave Technology Best Paper Award (Beijing, CHINA), the 2002 Georgia Institute of Technology-Electrical and Computer Engineering Outstanding Junior Faculty Award, the 2001 ACES Conference Best Paper Award, the 2000 National Science Foundation (NSF) CAREER Award, and the 1997 Best Paper Award of the International Hybrid Microelectronics and Packaging Society.

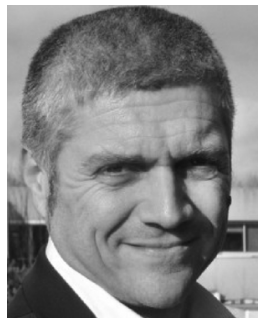

Robert Plana (M'98-SM'08) was born in Toulouse, France, in March 1964. He received the Ph.D. degree from the Laboratoire d'Analyse et d'Architecture des Systèmes (LAAS), National Center for Scientific Research (CNRS), Toulouse, France, and Paul Sabatier University, Toulouse, France, in 1993. His doctoral research concerned the noise modeling and characterization of advanced microwave devices (HEMTs, pseudomorphic HEMT (HEMTs), and HBTs) that include reliability.

In 1993, he became an Associate Professor with the LAAS-CNRS, where he began a new research area concerning the investigation of millimeter-wave capabilities of silicon-based technologies. More precisely, he has focused on the microwave and millimeter-wave properties of $\mathrm{SiGe}$ devices and their capabilities for low-noise circuits. In 1995, he began a new project concerning the improvement of passives on silicon through the use of MEMS technologies. In 1999, he became involved with SiGe Semiconductor, Ottawa, ON, Canada, where he was involved with low-power and low-noise integrated circuits for RF applications. Since 2000, he has been a Professor with Paul Sabatier University and the Institut Universitaire de France, forming a research team with LAAS-CNRS in the field of microsystems and nanosystems for RF and millimeter-wave communications. The team's main interests are on the technology, design, modeling, test, characterization, and reliability of RF MEMS for low-noise and high-power millimeter-wave applications and the development of the MEMS integrated circuit (IC) concept for smart microsystems. He has built a network of excellence in Europe in this field, "AMICOM," regrouping 25 research groups. In 2004, he became Deputy Director of the Information and Communication Department, CNRS Headquarters. From January 2005 to January 2006, he was the Director of the Information and Communication Department, CNRS. Since 2006, he has been heading a research group with LAAS-CNRS in the field of microsystems and nanosystems for wireless communications. From November 2007 to November 2009, he was with the "French Research Agency," where he was the Project Officer of the National Nanotechnology Initiative. Since November 2009, he has been the Head of the Physic, Mathematics, Nanosciences and Nanotechnology, Information and Communication Technology Department, Ministry of Research, where he is in charge of defining the French strategic for research and innovation. He has authored or coauthored over 300 international journals and conferences.

Dr. Plana was the recipient of a 1999 special award from CNRS for his works on silicon-based technologies for millimeter-wave communications. 